\title{
Novel Cylinder Movement Modeling Method Based on Aerodynamics
}

\author{
Jian-Qing Zhang ${ }^{1} \cdot$ Xiao-Mei Hu ${ }^{1} \cdot$ Jin-Sheng Kang ${ }^{2} \cdot$ Feng Xiong $^{1} \cdot$ \\ Ning Zeng ${ }^{1}$
}

Received: 4 December 2016/Revised: 13 April 2017/ Accepted: 11 July 2017/Published online: 20 July 2017

(C) Chinese Mechanical Engineering Society and Springer-Verlag GmbH Germany 2017

\begin{abstract}
The cylinder movement is affected by multiple factors and it is difficult to establish the accurate movement model of the cylinder. In order to improve the reliability of the production line design and to speed up the production line debugging, a novel cylinder movement modeling method based on aerodynamics is proposed. The kinetic theory, thermodynamic theory and kinematics knowledge are applied and integrated various factors which affect the movement characteristics of the cylinder are considered. According to the proposed mathematical model of cylinder movement, thecombined simulation software of cylinder movement based on Visual Studio and Visual Component (3D Create) is developed to calculate thevelocity, acceleration and movement time of the cylinders during the running of the assembly line. Comparison results of cylinder's movement time under different intake air and displacement show that the mathematical model of cylinder movement based on aerodynamic is more accurate and the degree of fittingis 0.9846 , which proves the effectiveness of the combined simulation software of cylinder movement. By the cylinder movement modeling method based on
\end{abstract}

Supported by National Natural Science Foundation of China (Grant No. 41101454), Shanghai Grand Science \& Technology Program of China (Grant No. 16111105900), and Shanghai Municipal

Commission of Economy and Informatization of China (Grant No. 160646).

Xiao-Mei Hu

sufeimasohxm@163.com

1 School of Mechatronic Engineering and Automation, Key Laboratory of Intelligent Manufacturing and Robotics, Shanghai University, Shanghai 200072, China

2 College of Engineering, Design and Physical Sciences, Brunel University London, Uxbridge UB8 3PH, UK aerodynamic, accurate value of takt and the debug parameters can be calculated as a reference for the designers and debuggers of the cylinder-driven assembly lines.

Keywords Aerodynamics - Thermodynamics · Cylinder characteristic $\cdot$ Combined simulation

\section{Introduction}

With the progress and development of science and technology, manufacture has been evolved into a complex engineering system composed of many manufacturing elements instead of a simple process of individual behavior and the isolated machine. Production line is an effective combination of man and machine, which has been widely used in the manufacturing industry. Along with the emergence of flexible manufacturing, agile manufacturing, JIT manufacturing, networked manufacturing, and so on, manufacturing technology began to develop in the direction of systematic, flexible, reconfigurable, integrated, networked, intelligent and greenregeneration. The transformation of manufacturing mode and the manufacturing technology also influences planning, design and operation of manufacturing system dramatically $[1,2]$.

Production line without manual operation can improve production efficiency and reduce labor costs greatly. An important indicator of production line is the takt that refers to the tact time required for producing a single product by the production line. The takt of production line is closely related to cylinder motion. In the design of production line, a virtual simulation is often carried out to verify whether it can meet the requirements of the takt of production line. 
For the cylinder-driven assembly line, an important part of virtual simulation of production line is to simulate the cylinder movement. Since there are a large number of cylinder components in the assembly line, each cylinder motion can affect the takt of assembly line in the virtual simulation. The movement time of the cylinder is determined by multiple factors, including load, friction, pressure, temperature, flow. Thus, it is critical to build the accurate movement model of cylinder and obtain the motion parameters under different conditions in order to accurately calculate the takt of assembly line.

There have been many studies about cylinder motion characteristics analysis. Guoliang Wu built an experimental platform to collect and analyze data, and obtained a polynomial relationship of cylinder friction and velocity. The minimum creep velocity of cylinder was drawn and the speed of cylinder was analyzed by the process results of displacement data [3]. Di Zhu and Sai Ye put forward a theoretical method to calculate the equivalent thermal power during the charging and discharging process of cylinder, which predicted the temperature changes of cylinder wall when cylinder reached the thermal steady state equilibrium [4]. Jingya Kuang studied the impact cushioning performance about the load changes, and established a mathematical model of buffer cylinder about energy, flow and movement based on aerodynamics [5]. Yukio Terashima mainly analyzed the cylinder's friction characteristics and researched the influence of friction on cylinder's movement [6]. Kawakami et al. did some research on dynamic characteristics of pneumatic cylinder [7]. Jun Huang and Xiaoning Li did dimensional analysis about creeping phenomena of cylinder by experiment [8]. However, the existing researches on the modeling of cylinder movement only focused on a very limited number of motion characteristics and contributing factor of the cylinder, and these methods do not constitute a comprehensive analysis of various factors on the cylinder movement characteristics.

Gas viscosity refers to the nature of the friction generated between the layers of the gas when the gas flows. Gas compressibility refers to the nature of gas volume reduction with the increase of pressure. Thermal conductivity is the performance of material conducting heat. These factors which impact the movement of cylinder should be considered in the establishment of the mathematical model of cylinder movement to improve the accuracy of the model. The energy loss of the cylinder during movement and the influence of gas compressibility on the cylinder motion characteristics are also regarded as effective factors to establish the mathematical model of the cylinder movement. Therefore, this paper takes into account of various impact factors (including friction, load, pressure, flow, heat loss, gas compression, etc.) on the cylinder motion characteristics (including velocity, acceleration, motion time), and aims to create a more inclusive and complete cylinder movement model. The mathematical model is introduced into the developed simulation software which is built by Visual Studio and Visual Component (3D Create), and combined simulation of the cylinder movement is achieved based on the simulation software. At the same time, simulation results are recorded. In comparison with the physical lab experimental data, the simulation accuracy on the cylinder movement is verified, which shows the effectiveness of the novel cylinder movement modeling method based on aerodynamics.

\section{Analysis on Impact Factors of Cylinder Movement}

Cylinder movement model consists of the models of velocity, acceleration and movement time of cylinder. Movement process of the cylinder is divided into four parts: start-up process, speed-up process, uniform process and stop process. Among them, start-up process includes two states: stationary state and critical state. Figure 1 shows all parameters in the conditions of stationary state, critical state, acceleration state and steady state.

In Figure 1, parameter instructions are as follows: $P_{s}$ is the pressure of gas source $(\mathrm{MPa}) ; P_{1}$ is the pressure of intake side in stationary state $(\mathrm{MPa}) ; P_{2}$ is the pressure of intake side in the critical state $(\mathrm{MPa}) ; P_{3}$ is the pressure of intake side in the acceleration state $(\mathrm{MPa}) ; P_{4}$ is the pressure of intake side in the steady state $(\mathrm{MPa}) ; P_{\mathrm{a}}$ is atmospheric pressure $(\mathrm{MPa}), P_{\mathrm{a}}=0.101 \mathrm{MPa} ; T_{s}$ is the temperature of intake pipe $(\mathrm{K}) ; T_{\mathrm{a}}$ is the outdoor temperature $(\mathrm{K}) ; f$ is the static friction $(\mathrm{N}) ; f_{1}$ is the maximum static friction $(\mathrm{N}) ; f_{2}$ is the kinetic friction force $(\mathrm{N})$; $m$ is the load of cylinder $(\mathrm{kg})$.

As can be seen from the above illustration, the pressure and temperature in the intake chamber vary with the movement of cylinder, so does the friction. However, gas properties in the intake side and outlet side remain unchanged.

\subsection{Cylinder's Internal Pressure}

When the cylinder is in the stationary state of the start-up process, the pressure in the cylinder intake side is represented as $P_{1}=P_{\mathrm{a}}$.

When the cylinder is in the critical state of the start-up process, according to the theory of piston force balance, the following formula can be obtained:

$P_{2} S=f_{1}+P_{\mathrm{a}} S$.

In Eq. (1), $P_{\mathrm{a}}$ is the atmospheric pressure $(\mathrm{MPa}) ; S$ is the cross-sectional area of the cylinder $\left(\mathrm{mm}^{2}\right)$. In this case, the intake side pressure is calculated by 


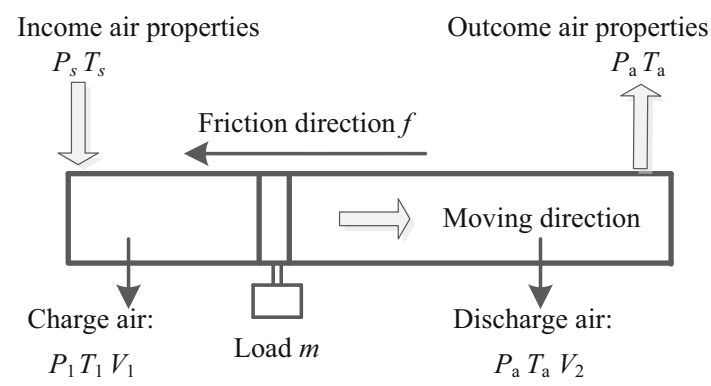

(a) Start-up process (stationary state)

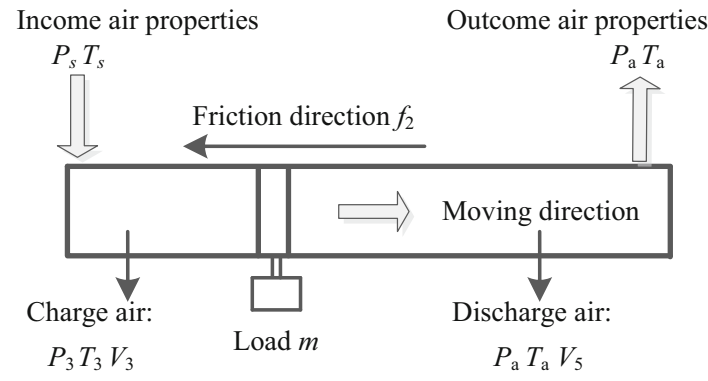

(c) Speed-up process (acceleration state)

Figure 1 Parameter statement in each cylinder movement process

$P_{2}=\left(f_{1}+P_{\mathrm{a}} S\right) / S$.

Similarly, when the cylinder is in the steady state, the intake-side pressure is calculated by

$P_{4}=\left(f_{2}+P_{\mathrm{a}} S\right) / S$.

\subsection{Thermodynamic Analysis of Gas in Side Cylinder}

According to modern practical pneumatic technology, first law of thermodynamics and mass and heat capacity formula can be obtained as [9]

$\delta q=\mathrm{d} I+P \mathrm{~d} V$,

$c=\delta q / \mathrm{d} T$.

In Eq. (4) and Eq. (5), $q$ is heat (J), $I$ is gas internal energy (J), $P$ is gas pressure (MPa), $V$ is volume $\left(\mathrm{dm}^{3}\right), c$ is heat capacity of mass $(\mathrm{J} / \mathrm{kg} \cdot \mathrm{K}), T$ is temperature $(\mathrm{K})$. In the process of cylinder motion, the parameters, such as $P, T$ and $V$, vary because cylinder is not adiabatic with the outside. In this case, the gas in the cylinder is the changeable process. According to the first law of thermodynamics and mass and heat capacity formula, it can be seen that:

$\delta q=c \mathrm{~d} T=c_{V} \mathrm{~d} T+P \mathrm{~d} V$.

In Eq. (6), $c_{V}$ is quality volumetric heat capacity $(\mathrm{J} / \mathrm{kg} \cdot \mathrm{K})$. By differentiating state equation $P V=R T$ and substituting

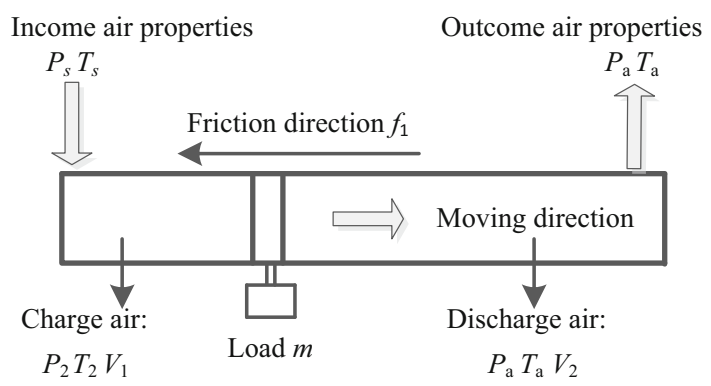

(b) Start-up process (critical state)

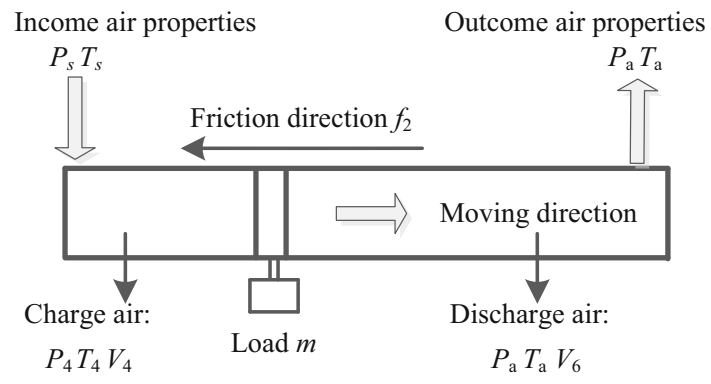

(d) Uniform process (steady state)

the result into Eq. (6), state equation of variable processcan be obtained as

$P V^{n}=C$.

In Eq. (7), $C$ is a constant. When $n=0, P V^{0}=P=C$ is isobaric process; when $n=1, P V=C$ is isothermal process; when $n= \pm \infty, V=C$ is isochoric process.

\subsection{Cylinder Throttle Valve Control}

Flow is defined as the amount of fluid per unit time through a cross-sectional area. The volume flow is used to incompressible fluid, but only mass flow is used to compressible fluid [9]. When the gas passes through the throttle, the entire flow is a stable one-dimensional flow, which is similar to the case of the shrinking nozzle. Therefore, the gas through the throttle can be calculated by the mass flow equation of the shrinking nozzle [10]. The empirical formula is shown as follows:

$Q_{m}=\frac{\mathrm{d} M}{\mathrm{~d} t}=\frac{C_{q} \sqrt{2 k} A P_{s} \varphi_{(\sigma)}}{\sqrt{R T_{s}(k-1)}}$,

$\varphi_{(\sigma)}=\left\{\begin{array}{cc}\left(\frac{2}{k+1}\right)^{\frac{k}{k-1} \sqrt{\frac{k-1}{k+1}},} & \sigma \leq C_{t} ; \\ \sqrt{\sigma^{\frac{2}{k}}-\sigma^{\frac{k+1}{k}}}, & \sigma>C_{t} ;\end{array}\right.$ 
$\sigma=\frac{P_{\mathrm{s}}}{P_{\mathrm{i}}}$

$C_{t}=\left(\frac{2}{k+1}\right)^{\frac{k}{k-1}}=0.528$.

In Eqs. (8)-(11), $Q_{m}$ is the mass flow $(\mathrm{kg} / \mathrm{s}) ; M$ is gas mass $(\mathrm{kg}) ; \quad R$ is gas constant, for atmosphere, $R=287 \mathrm{~N} \cdot \mathrm{m} /(\mathrm{kg} \cdot \mathrm{K}) ; t$ is the time $(\mathrm{s}) ; A$ is the throttle area $\left(\mathrm{mm}^{2}\right) ; C_{q}$ is the flow coefficient, and $C_{q}=0.95-0.98 ; k$ is adiabatic coefficient, and $k=1.4 ; C_{t}$ is the critical pressure ratio; $P_{\mathrm{i}}$ is the intake side pressure under different states (MPa). In the process of the cylinder motion, the throttle upstream pressure is larger than the throttle downstream pressure, namely, $P_{\mathrm{s}}>P_{\mathrm{i}}$, $\sigma>0.528$. In this case, the gas mass flow through the throttle is calculated as:

$Q_{m 1}=\frac{\mathrm{d} M}{\mathrm{~d} t}=\frac{1.55 C_{q} A P_{s} \sqrt{\left(\frac{P_{s}}{P_{1}}\right)^{1.43}-\left(\frac{P_{s}}{P_{1}}\right)^{1.71}}}{\sqrt{0.4 R T_{s}}}$

In Eq. (12), $Q_{m 1}$ is gas mass flow (kg/s). When the cylinder is in equilibrium state, the gas mass flow through the throttle is defined as:

$Q_{m 3}=\frac{\mathrm{d} M}{\mathrm{~d} t}=\frac{1.55 C_{q} A P_{s} \sqrt{\left(\frac{P_{s}}{P_{3}}\right)^{1.43}-\left(\frac{P_{s}}{P_{3}}\right)^{1.71}}}{\sqrt{0.4 R T_{s}}}$

In Eq. (13), $Q_{m 3}$ is gas mass flow in equilibrium state $(\mathrm{kg} / \mathrm{s})$

\subsection{Gas Compressibility during Cylinder Movement}

The impact of gas compressibility on the cylinder motion is mainly in the startup process. In the start-up process, it has a certain time for the gas to fill the entire space in the intake side until the pressure reaches $P_{2}$. During this filling process, according to the law of mass conservation, the following formula can be obtained:

$\rho_{2} V_{1}=\rho_{1} V_{1}+\rho \Delta V$

Where $\rho_{2}$ is the intake side density after filling $(\mathrm{g} / \mathrm{mL}) ; \rho_{1}$ is the intake-side air density in the initial state $(\mathrm{g} / \mathrm{mL})$, $\rho_{1}=1.29 \mathrm{~kg} / \mathrm{m}^{3} ; \rho$ is gas density of the intake pipe $(\mathrm{g} /$ $\mathrm{mL}) ; \Delta V$ is volume of gas which gets into the intake side from the throttle valve $(\mathrm{mL})$.

In the whole start-up process, the cylinder's temperature is assumed to be constant. Then the start-up process belongs to adiabatic process, and $\rho \propto P$ is satisfied. According to Eq. (14), the following formula can be obtained:
$\Delta V_{1}=\frac{\left(\rho_{2}-\rho_{1}\right) V_{1}}{\rho}=\frac{\left(P_{2}-P_{1}\right) V_{1}}{P_{S}}$

In Eq. (15), $\Delta V_{1}$ is volume change amount caused by gas compressibility $(\mathrm{mL})$. Because the volume of gas getting into the cylinder is equal to the volume of gas getting out of the cylinder, the following expression is shown:

$\Delta V_{1}=Q_{v 1} t_{1}$.

In Eq. (16), $Q_{v 1}$ is the gas flow through the throttle in the start-up process $(\mathrm{mL} / \mathrm{s})$. In the start-up process, the gas flow through the throttle is

$Q_{v 1}=\frac{1.55 C_{q} A P_{s} \sqrt{\left(\frac{P_{s}}{P_{\mathrm{a}}}\right)^{1.43}-\left(\frac{P_{s}}{P_{\mathrm{a}}}\right)^{1.71}}}{\rho_{1} \sqrt{0.4 R T_{s}}}$.

According to Eq. (15)-Eq. (17), the start time can be calculated as

$t_{1}=\frac{\left(P_{2}-P_{1}\right) V_{1} \rho_{1} \sqrt{0.4 R T_{s}}}{1.55 C_{q} A P_{s}^{2} \sqrt{\left(\frac{P_{s}}{P_{\mathrm{a}}}\right)^{1.43}-\left(\frac{P_{s}}{P_{\mathrm{a}}}\right)^{1.71}}}$.

\subsection{Gaseous Heat Loss during Cylinder Movement}

Since the gas in the cylinder is compressed, the temperature of gas in the cylinder rises. At this time, temperature gradient generates between the gas inside the cylinder and the gas outside the cylinder, which can cause heat loss. The heat loss of the gas is equivalent to the volume change amount of gas at certain temperature according to the conductive formula of gas:

$\Delta q=-\lambda \frac{\partial T}{\partial s}=-\lambda \frac{T_{1}-T_{a}}{s}$.

where $\Delta q$ is the heat loss $(\mathrm{J}), \frac{\partial T}{\partial s}$ is the temperature gradient $(\mathrm{K} / \mathrm{m}), \lambda$ is the thermal conductivity $(\mathrm{W} /(\mathrm{m} \cdot \mathrm{K}))$, the $\lambda$ of air is $2.47 \times 10^{-2} \mathrm{~W} /(\mathrm{m} \cdot \mathrm{K}), s$ is the thickness of cylinder wall (m). When the temperature gradient is small, the influence of the gas thermal conductivity can be ignored. Any gas has various properties as described above. By the laws of thermodynamics, according to Eq. (7), heat loss amount on account of the reduction of the gas volume at constant temperature can be defined as follows:

$\Delta q=c_{1} \rho_{1} \Delta V T_{1}$.

where $c_{1}$ is the specific heat capacity of air, $c_{1}=1 \times 10^{3}$ $\mathrm{J} / \mathrm{kg} \cdot{ }^{\circ} \mathrm{C}, \Delta V$ is the change amount of gas volume $\left(\mathrm{m}^{3}\right)$. According to Eq. (19) and Eq. (20), the change volume of gas can be obtained:

$\Delta V_{2}=\frac{\lambda\left(T_{1}-T_{2}\right)}{s c_{1} \rho_{1} T_{1}}$ 
In Eq. (21), $\Delta V_{2}$ is volume change amount caused by gaseous heat loss $(\mathrm{mL})$.

Similarly, according to Eq. (13) and Eq. (21), the cylinder movement time influenced by the heat loss of gas is calculated as

$$
t_{4}=\frac{\Delta V_{2}}{Q_{v 3}}=\frac{\lambda\left(T_{1}-T_{2}\right) \sqrt{0.4 R T_{s}}}{1.55 s c_{1} \rho_{1} T_{1} C_{q} A P_{s} \sqrt{\left(\frac{P_{s}}{P_{3}}\right)^{1.43}-\left(\frac{P_{s}}{P_{3}}\right)^{1.71}}} .
$$

\section{Mathematical Model of Cylinder Movement}

\subsection{Velocity Modeling of Cylinder Movement}

Many factors affect the velocity of cylinder movement, such as cylinder's diameter, flow of solenoid valve, diameter and length of the gas pipes, the supply amount of gas sources, and the position of the control components in the gas path [11]. In this paper, the cylinders use intake throttling way and cylinder's diameter, flow of solenoid valve and the supply amount of gas sources are mainly considered.

In the cylinder'sstart-up process, the velocity of the cylinder $v_{1}=0$. In the cylinder's uniform process, the intake side pressure is $P_{3}$, the volume flow of throttle valve is $Q_{v 3}$, volume increment of the cylinder intake sideis equal to the intake air. So the following formula can be obtained:

$Q_{v 3}=\frac{Q_{m 3}}{\rho_{3}}=S \cdot v_{2}$.

According to Eq. (13) and Eq. (23), the velocity of cylinder is calculated as follows:

$$
v_{2}=\frac{1.55 C_{q} A P_{s} \sqrt{\left(\frac{S P_{s}}{f_{2}+P_{\mathrm{a}} S}\right)^{1.43}-\left(\frac{S P_{s}}{f_{2}+P_{\mathrm{a}} S}\right)^{1.71}}}{\rho_{3} S \sqrt{0.4 R T_{s}}} .
$$

In the cylinder'sspeed-up process, thevelocity of cylinder increases from zero to maximum value $v_{2}$ in uniformly accelerated motion.

\subsection{Acceleration Modeling of Cylinder Motion}

From the stationary state to the steady state, the cylinder has a process of variable accelerated motion. During this process, the pressure of the intake side fluctuates between $P_{2}$ and $P_{4}$, and the fluctuation curve is shown in Figure 2.

In the cylinder'sspeed-up process, the creeping phenomenon is generated because of the impact of instable pressure in the intake side and other factors. In order to facilitate the calculation, the pressure of intake side inthe

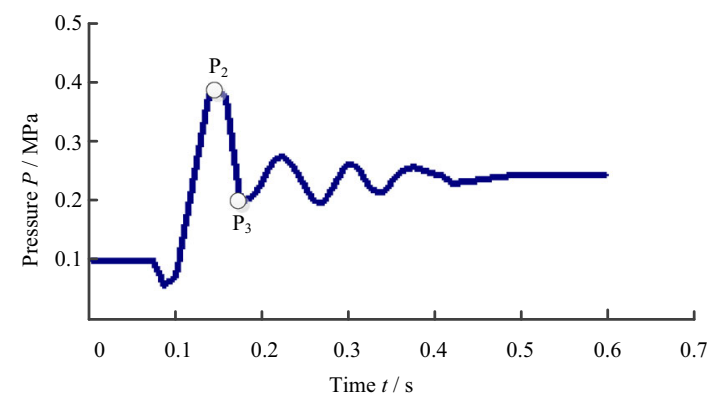

Figure 2 Pressure change in the cylinder's intake sidein the process of variable accelerated motion

cylinder's speed-up process is equivalent to a constant, which can be expressed as

$P_{3}=\left(P_{2}+P_{4}\right) / 2=\left(f_{1}+f_{2}+2 P_{\mathrm{a}} S\right) / 2 S$.

Therefore, the cylinder's acceleration $a$ in the speed-up process is calculated as

$a=\frac{\left(P_{2}+P_{4}\right) S}{2 m}=\frac{f_{1}+f_{2}+2 P_{\mathrm{a}} S}{2 m}$.

\subsection{Movement Time Modeling of Cylinder}

Motion time of the cylinder consists of three parts: the start-up time $t_{1}$, acceleration time $t_{2}$ and uniform motion time $t_{3}$.

In the start-up process, the gas gets into cylinder's intake side from intake pipe quickly, which makes the intake-side pressure $P_{\mathrm{a}}$ increase to $P_{2}$. By calculation, the cylinder's start-up time $t_{1}$ in this process can be obtained.

In the cylinder's speed-up process, according to Newton's laws of motion:

$v_{2}=a t_{2}$,

$l_{1}=\frac{v^{2}}{2 a}$,

$v_{2} t_{3}=l-l_{1}=l_{2}$.

In Eqs. (27)-(29), $v_{2}$ is the cylinder's velocity in steady state $(\mathrm{mm} / \mathrm{s}), a$ is cylinder's acceleration $\left(\mathrm{mm} / \mathrm{s}^{2}\right), t_{2}$ is cylinder's acceleration time (s), $l_{1}$ is cylinder's accelerated motion displacement $(\mathrm{mm}), l_{2}$ is cylinder's uniform motion displacement $(\mathrm{mm}), t_{3}$ is cylinder's motion time in steady state $(\mathrm{s}), l$ is cylinder's stroke $(\mathrm{mm})$.

According to Eq. (24), Eq. (26) and Eq. (27), cylinder's acceleration time can be obtained as follows:

$t_{2}=\frac{3.1 m C_{q} A P_{s} \sqrt{\left(\frac{S P_{s}}{f_{2}+P_{\mathrm{a}} S}\right)^{1.43}-\left(\frac{S P_{s}}{f_{2}+P_{\mathrm{a}} S}\right)^{1.71}}}{\rho_{3} S\left(f_{1}+f_{2}+2 P_{\mathrm{a}} S\right) \sqrt{0.4 R T_{s}}}$. 
According to Eq. (24), Eq. (26) and Eq. (28), cylinder's displacement in speed-up process can be obtained as follows:

$$
l_{1}=\frac{2.4 m C_{q}^{2} A^{2} P_{s}^{2}\left[\left(\frac{S P_{s}}{f_{2}+P_{\mathrm{a}} S}\right)^{1.43}-\left(\frac{S P_{s}}{f_{2}+P_{\mathrm{a}} S}\right)^{1.71}\right]}{0.4\left(f_{1}+f_{2}+2 P_{\mathrm{a}} S\right) R T_{s} \rho_{3}^{2} S^{2}} .
$$

According to Eq. (24), Eq. (29) and Eq. (31), cylinder's uniform motion time can be obtained as follows:

$$
\begin{gathered}
t_{3}=\frac{l \rho_{3} S \sqrt{0.4 R T_{s}}}{1.55 C_{q} A P_{s} \sqrt{\left(\frac{S P_{s}}{f_{2}+P_{\mathrm{a}} S}\right)^{1.43}-\left(\frac{S P_{s}}{f_{2}+P_{\mathrm{a}} S}\right)^{1.71}}} \\
-\frac{1.55 m C_{q} A P_{s} \sqrt{\left(\frac{S P_{s}}{f_{2}+P_{\mathrm{a}} S}\right)^{1.43}-\left(\frac{S P_{s}}{f_{2}+P_{\mathrm{a}} S}\right)^{1.71}}}{\sqrt{0.4 R T_{s}}\left(f_{1}+f_{2}+2 P_{\mathrm{a}} S\right) \rho_{3} S} .
\end{gathered}
$$

\section{Combined Simulation Based on Visual Component and Visual Studio}

The processes of cylinder movement combined simulation based on Visual Component (VC) and Visual Studio (VS) are as follows:

(1) Set up cylinder model in the simulation module based on Visual Component, including module structure, parameter setting, system running and other steps;

(2) Create a simulation operation interface in Visual Studio, which contains the input and output of parameters, cylinder type, and so on, use C\# language programming for the backstage setting;

(3) Import the mathematical model of cylinder movement into the Visual Studio, and run the program according to the formula in Sect. 3.3;

(4) Output the calculation results of the mathematical model of the cylinder motion in Visual Studio to Visual Component so as to realize the combined simulation of Visual Component and Visual Studio.

Figure 3 is a combined simulation flow chart based on Visual Component and Visual Studio. Through the interface, air pressure, air inflow, inertia load and cylinder type, speed, acceleration, displacement and other parameters are inputted or outputted to realize the interaction with the cylinder simulation system which contains a variety of models of cylinders.

Three-dimensional models of cylinder standard partscan be established with the same size through software Pro/E. By converting the format using 3Dmax software, the 3D models of cylinder standard parts are imported and shown

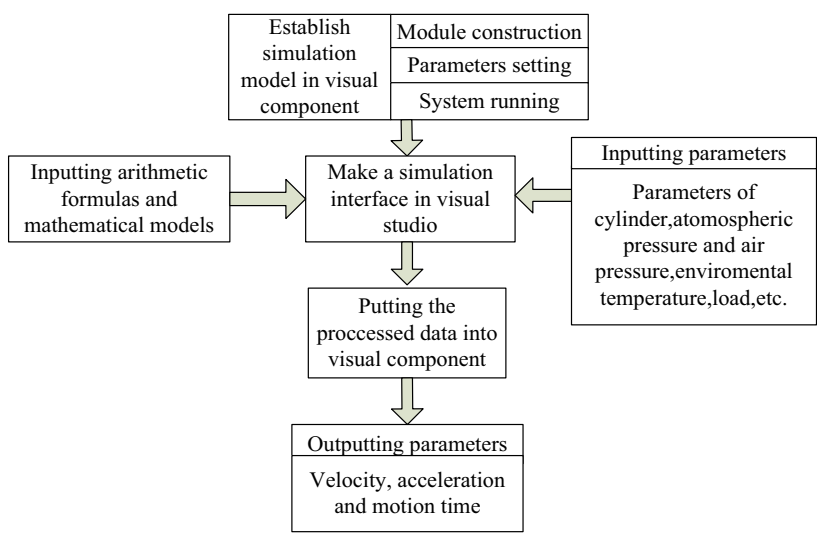

Figure 3 Flowchart of cylinder combined simulation based on VC and VS

into the 3D visualization interface based on Visual Component software, which is shown in Figure 4.

\section{Combined Simulation Result Analysis}

In order to verify the reliability of combined simulation results and the accuracy of mathematical model of cylinder motion, test platform was constructed and the velocity, acceleration and movement time were obtained in the process of actual experiment. Comparative analysis on the experimental results and simulation results has been carried out.

\subsection{Construction of Test Platform and Collection of Experimental Results}

In the experiment, the cylinder model adopts MY3B16-700 (SMC), throttle value type is SRCT-03, work pressure is $0.6 \mathrm{MPa}$, and displacement sensors, pressure sensors, directional control valve, flow control value, filters, oscillography, air source are used in the test platform [12-16]. The types and precisions of measuring apparatuses are shown in Table 1. Figure 5 is the experimental model of cylinder motion.

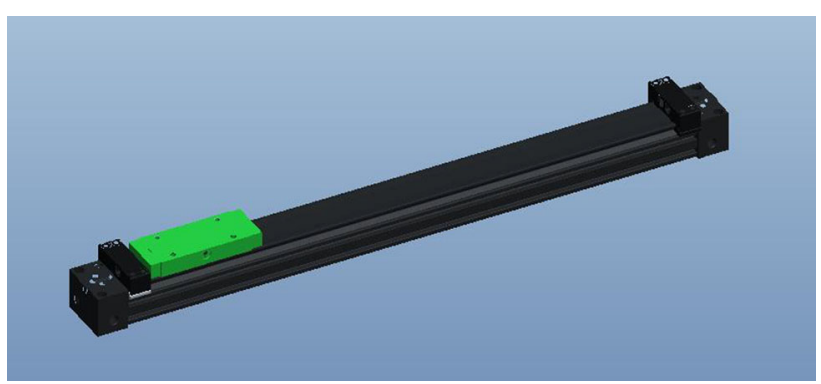

Figure 4 3D visualization interface based on Visual Component 
In order to ensure the consistency of simulation conditions with the experimental conditions of the cylinder motion, the parameters of test platformare set in Table 2. In Table 2, the max static friction force depends on the size of different cylinders. Different types of cylinders have different max static friction force. In the purchase of cylinders, the max static friction force can be obtained from the product brochures. In this paper, $0.25 \mathrm{~N}$ is obtained from the cylinder parameters in the specification of cylinder we selected. The sliding friction force has the direction along the contact surface and opposite to the direction of relative motion of the cylinder. It was calculated by the formula $f_{2}=u F_{n}$. Where, $F_{n}$ is the pressure on the cylinder piston surface, $u$ is the dynamic frictional coefficient, which is provided by the cylinder manufacturer in the specification.

\subsection{Comparison of Experimental Results and Simulation Results}

Table 3 and Table 4 show the comparison result of experimental data and simulation data. Comparison of cylinder's movement time under different intake air is shown in Table 3. Comparison of cylinder's movement time under different displacement is shown in Table 4. The accuracy of the combined simulation is verified by comparison with the experimental results and the cylinder motion simulation resultsbased on dimensional analysis. Cylinder motion simulation method based on dimensional analysis uses the principle of dimensional analysis to get the cylinder dimensionless mathematical model [17].

Figure 6 is the comparison of cylinder's movement time under different intake air. The blue curve, the red curve and the green curve correspond to the cylinder's running time in the testing experiment, the combined simulation running time and the cylinder's running time based on dimensional analysis. The method based on dimensional analysis adopts dimensional analysis theory to make mathematical model dimensionless and get a dimensionless model, and the simulation curve can be obtained by using MATLAB eventually [18-20].

Table 1 Types and precisions of measuring apparatuses

\begin{tabular}{lll}
\hline Measuring apparatus & Type & Precision \\
\hline Pressure sensor & PSE540A-R06 & $0.1-1 \mathrm{MPa}$ \\
Temperature sensor & Type T thermocouple & $1{ }^{\circ} \mathrm{C}$ \\
Displacement sensor & LK-G500 & $700 \mathrm{~mm}, 0.01 \mathrm{~mm}$ \\
Data collection & NI-6259 & - \\
\hline
\end{tabular}

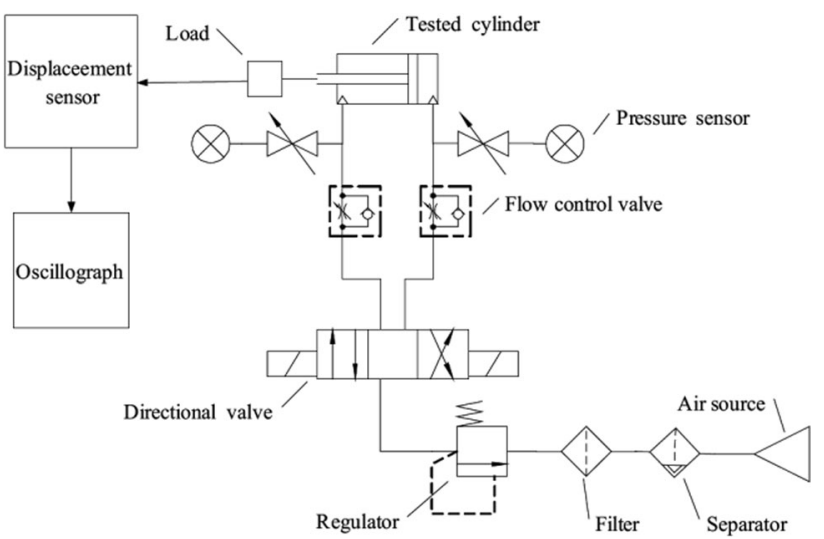

Figure 5 Experimental model of cylinder motion

Table 2 Parameter setting of cylinder movement test platform

\begin{tabular}{ll}
\hline Parameter & Value \\
\hline Displacement $l / \mathrm{mm}$ & 700 \\
Diameter $D / \mathrm{mm}$ & 16 \\
Sectional area of throttle $S / \mathrm{mm}^{2}$ & 1.2 \\
Pressure of gas source $P_{\mathrm{s}} / \mathrm{MPa}$ & 0.6 \\
Atmospheric pressure $P_{\mathrm{a}} / \mathrm{MPa}$ & 0.101 \\
Load $m / \mathrm{kg}$ & 2 \\
Static friction $f_{1} / \mathrm{N}$ & 0.25 \\
Kinetic friction $f_{2} / \mathrm{N}$ & 0.2 \\
Cylinder's sectional area $S / \mathrm{mm}^{2}$ & 201 \\
Indoor temperature $T_{\mathrm{a}} /{ }^{\circ} \mathrm{C}$ & 23 \\
Temperature in uniform motion $T_{4} /{ }^{\circ} \mathrm{C}$ & 31 \\
Thickness of cylinder $E / \mathrm{mm}$ & 1 \\
\hline
\end{tabular}

Table 3 Comparison of cylinder's movement time under different intake air

\begin{tabular}{|c|c|c|c|c|}
\hline \multirow{2}{*}{$\begin{array}{l}\text { Experiment } \\
\text { No. }\end{array}$} & \multirow{2}{*}{$\begin{array}{l}\text { Intake air } \\
I_{A} /\left(\mathrm{mL} \cdot \mathrm{s}^{-1}\right)\end{array}$} & \multicolumn{3}{|l|}{ Time $t / \mathrm{s}$} \\
\hline & & Testing & $\begin{array}{l}\text { Combined } \\
\text { simulation }\end{array}$ & $\begin{array}{l}\text { Based on } \\
\text { dimensional } \\
\text { analysis }\end{array}$ \\
\hline 1 & 0.2 & 15 & 15.2 & 11 \\
\hline 2 & 0.5 & 12 & 12.2 & 10 \\
\hline 3 & 1 & 11 & 10.8 & 9 \\
\hline 4 & 2 & 9 & 8.8 & 8 \\
\hline 5 & 3 & 6 & 6.2 & 5.9 \\
\hline 6 & 4 & 3.9 & 3.7 & 3.5 \\
\hline 7 & 5 & 3 & 3.2 & 3 \\
\hline 8 & 6 & 2.4 & 2.2 & 2 \\
\hline 9 & 7 & 1.8 & 2 & 1.8 \\
\hline 10 & 8 & 1.5 & 1.6 & 1.5 \\
\hline 11 & 9 & 1.2 & 1.1 & 1 \\
\hline
\end{tabular}


Table 4 Comparison of cylinder's movement time under different displacement

\begin{tabular}{|c|c|c|c|c|}
\hline \multirow{2}{*}{$\begin{array}{l}\text { Experiment } \\
\text { No. }\end{array}$} & \multirow{2}{*}{$\begin{array}{l}\text { Displacement } \\
D_{P} / \mathrm{mm}\end{array}$} & \multicolumn{3}{|l|}{ Time $t / \mathrm{s}$} \\
\hline & & Testing & $\begin{array}{l}\text { Combined } \\
\text { simulation }\end{array}$ & $\begin{array}{l}\text { Based on } \\
\text { dimensional } \\
\text { analysis }\end{array}$ \\
\hline 1 & 0 & 0.4 & 0.4 & 0 \\
\hline 2 & 100 & 1.21 & 1.2 & 1 \\
\hline 3 & 200 & 3 & 3.1 & 2.8 \\
\hline 4 & 300 & 4 & 3.9 & 3.3 \\
\hline 5 & 400 & 4.7 & 4.72 & 4 \\
\hline 6 & 500 & 5.4 & 5.3 & 4.4 \\
\hline 7 & 600 & 6.1 & 6.05 & 5.1 \\
\hline 8 & 700 & 7 & 6.95 & 5.8 \\
\hline
\end{tabular}

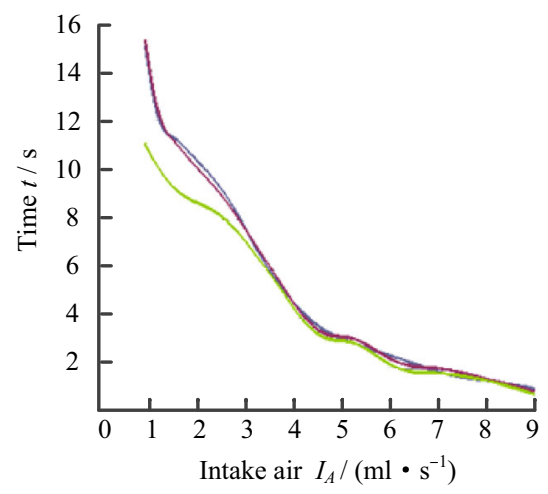

Figure 6 Comparison of cylinder's movement time under different intake air

Apparently, cylinder's actual movement time and combined simulation running time fit basically under different intake air. By calculation, its degree of fitting $R^{2}=0.9846$, which proves thatthe mathematical model of the cylinder motion combined simulation is very accurate. However, the impact factors of the cylinder motion characteristics are not considered, so the cylinder's simulation time based on dimensional analysis is significantly shorter than the actual cylinder operation and combined simulation running time. Moreover, the smaller intake air amount is, the larger the difference of the cylinder's simulation time based on dimensional analysis and the actual cylinder operation time is, which means the impact factors have a greater impact under the condition of smaller intake air amount.

Figure 7 is the comparison of cylinder's movement time under different displacement. The blue curve, the red curve and the green curve correspond to the cylinder's running time in the testing experiment, the combined simulation running time and the cylinder's running time based on dimensional analysis. From Figure 7, the start time of

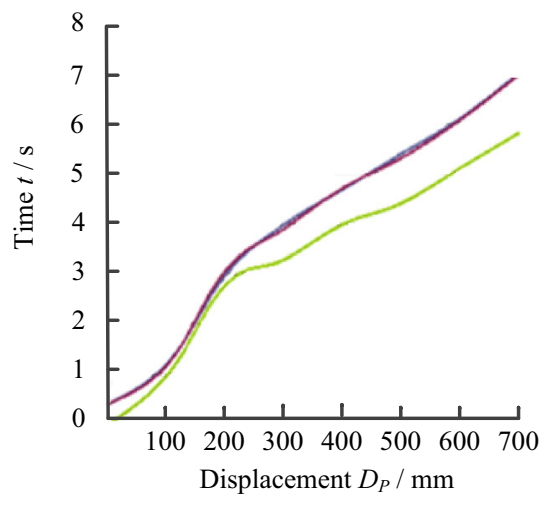

Figure 7 Comparison of cylinder's movement time under different displacement

cylinder simulation based on dimensional analysis is 0 and there is no reaction time. The actual experiment and combined simulation both have reaction time, and the reaction time is about $0.4 \mathrm{~s}$, and the reaction time decreases with the increase ofthe work pressure. In the subsequent operation process, cylinder's running time without considering aerodynamic is lower than the actual cylinder movement time and combined simulation running time, and the time gap continue increasing with the increase of displacement. This is because the gas viscosity resistance acts against the cylinder and the heat loss during the cylinder operation lead to the increase of cylinder movement time [21-27].

From Figure 7, cylinder motion combined simulation has more accurate results compared with the cylinder's simulation based on dimensional analysis, which proves the effectiveness of the mathematical model of cylinder movement.

\section{Conclusions}

(1) A novel, more inclusive mathematical modelling method on cylinder movement is proposed, which takes into account not only the friction, the inertia load on the cylinder motion characteristics, but also the impact of the gas compressibility, thermal conductivity, energy loss, viscosity and other aerodynamic and thermodynamic characteristics.

(2) A combined simulation software based on Visual Component (VC) and Visual Studio (VS) is developed, which integrated the above mathematical model, and can run the virtual simulation of cylinder movement by setting up the input parameters and can obtain the simulation results about cylinder movement.

(3) A physical lab testing platform with precision measuring apparatuses is built, which can measure 
the cylinder's motion under different loading conditions.

(4) The real world experimental results on the above platform show very good agreement with the combined simulation results under different conditions, with the degree of fitting $R^{2}=0.9846$, which proves that the mathematical model of the cylinder movement combined simulation is very accurate.

(5) The real world experimental results and combined simulation results are compared with the traditional mathematical model based on dimensional analysis theory, and it shows that the proposed method is much more accurate than dimensional analysis model.

\section{References}

1. X L Liu, G H He, F G Yan, et al. Large chip production mechanism under the extreme load cutting conditions. Chinese Journal of Mechanical Engineering, 2015, 28(2): 343-352.

2. K Naeem, M Ullah, A Tariq, et al. Optimization of steel bar manufacturing process using six sigma. Chinese Journal of Mechanical Engineering, 2016, 29(2):332-341.

3. G L Wu. Research on cylinder's comprehensive performance test system. Zhejiang: Zhejiang University Press, 2007.(in Chinese)

4. D Zhu, S Ye. Research on the temperature prediction of cylinder wall. Hydraulic and Pneumatic, 2015, 1(1):104-108.

5. J Y Kuang. The research of load affecting the buffering performance of the cylinder. Liaoning: Dalian Maritime University Press, 2012. (in Chinese)

6. T Yukio, K Yukio, K Sunao. A Study on the effects of friction characteristics in pneumatic cylinder. Proceedings of the Fourth International Symposium on Fluid Power Transmission and Control, Wuhan, China, April 8-10, 2003: 252-357.

7. Y Kawakami, J Akao, S Kawai, et al. some considerations on the dynamic characteristics of pneumatic cylinders. The Journal of Fluid Control, 1988, 19(2): 22-36.

8. J Huang, X N Li. Stick-slip criterion of cylinder based on experiment and dimensional analysis. Journal of Nanjing University of Science and Technology (Natural Science), 2006, 30(3): 279-284.

9. SMC (China) Company Limited. Modernpracticalpneumatic technology. Beijing: China Machine Press, 2008. (in Chinese)

10. Z W Cui. The modeling and simulation of cylinder's low friction characteristics. Heilongjiang: Harbin Institute of Technology Press, 2008. (in Chinese)

11. X T Li, W Si. Cylinder's speed control. Rubber Technology and Assembly, 2003, 29(8): 26-27.

12. L R Tokashiki, T Fujita, T Kagawa. Stick-slip Motion in pneumatic cylinders driven by mete: outer circuit (2nd report occurrence conditions). Transactions of the Japan Hydraulics \& Pneumatics Society, 2000, 31(6): 20-25.

13. G Belforte, G Mattiazzo, S Mauro. Measurement of friction force in pneumatic cylinders. Tribofest Journal, 2003, 20(2): 34-38.

14. D S Kim, S H Lee. The analysis of optimum design of cushioning pneumatic cylinder. Proceedings of the $3^{\text {rd }}$ International Symposium on Fluid Power Transmission and Control,Harbin, China, September 7-9, 1999: 421-425.
15. S L Cheng, H F Wang, W B Lu. The modeling and simulation of cylinder's dynamic system based on dimensional analysis. Chinese Hydraulics \& Pneumatics, 2008, 7(5): 36-41.

16. Y T Wang, R Singh. Computer simulation of a shock-absorbing pneumatic cylinder. Journal of Sound and Vibration, 1985, 93(3): 353-364.

17. S L Cheng, H F Wang, W B Lu. Modeling and simulating based on dimensional analysis for pneumatic cylinder dynamics system. Flow Measurement and Instrumentation, 2008, 20(7): 10-13.

18. D K M Arguelles, O J M Femandez, M E Blanco. Cylindrical three-hole pressure probe calibration for large angular range. Flow Measurement and Instrumentation, 2008, 20(2): 57-68.

19. D K M Arguelles, O J M Femandez, M E Blanco. Extended angular range of a three-hole cobra pressure probe for incompressible flow. Transactions of the ASME-Journal of Fluids Engineering, 2008, 130(10): 1-6.

20. Z G Xie, G L Tao. Study on cylinders at very low velocities. Huazhong University of Science and Technology, 2003, 4(5): 358-361.

21. B H Zhang, H F Cheng. Graph modeling and simulation of stickslip characteristics of a pneumatic system. The Sixth International Conference on Fluid Transmission and Control, Hangzhou, China, April 6-8, 2005: 65-71.

22. A Bakali, Q Olivier, R R Vaney. Design of components libraries for the transient simulation of an automotive refrigerant loop. Institution of Mechanical Engineers, 2003, 51(1): 611-620.

23. M F Perez-Pole, M Perez-Molina, J G Chica. Analysis of the state equations of a real gas at high pressures with the virial coefficients obtained from controlled chaotic oscillations. Chemical Engineering Science, 2016, 155(11): 482-503.

24. W Bao, H Zhou, G L Tao. A nonlinear controller for pneumatic cylinder cushion. Proceedings of the Fourth International Symposium on Fluid Power Transmission and Control, Wuhan, China, April 8-10, 2003: 370-373.

25. Z M Jing, B H Zhang, T Wang. Modeling and simulation of the characteristics of pneumatic cushion cylinders. Journal of Beijing Institute of Technology, 2002, 11(2): 129-132.

26. J Wang, D J D Wang, P R Moore. Modeling study analysis and robust servo control of pneumatic cylinder actuator system. Control Theory and Applications, 2001,148(1): 25-42.

27. W G Li, G L Tao. Modeling cylinder controlled by servo valve of hydraulic looper in hot strip mill. 2015 Chinese Automation Congress, Wuhan, China, November 27-29, 2015: 102-107

Jian-Qing Zhang, born in 1975, is currently a Ph.D. candidate at School of Mechatronic Engineering and Automation, Shanghai University, China. He received his master degree from China-Europe International Business School, China, in 2008. His research interests include E-commerce and internet communication.

Xiao-Mei Hu, born in 1978, is currently an associate professor at School of Mechatronic Engineering and Automation, Shanghai University, China. She received her Ph.D. degree on computer science and technology from Northwestern Polytechnical University, China, in 2007. She received her master degree on control theory and control engineering from Southwest University of Science and Technology, China, in 2003. She received her bachelor degree on computer science and technology from University of Electronic Science and Technology, China, in 2000. Her research interests include virtual reality, augment reality, intelligent manufacturing and visualization.

Jin-Sheng Kang, born in 1952, is currently a senior lecturer at Brunel University London, $U K$. $\mathrm{He}$ received his Ph.D. degree from 
Bournemouth University, UK, in 2001. Hisresearch interests include human modelling and simulation, CAD, industrial design.

Feng Xiong, born in 1974, is currently an associate professor at School of Mechatronic Engineering and Automation, Shanghai University, China.
Ning Zeng, born in 1991, is currently a master at School of Mechatronic Engineering and Automation, Shanghai University, China. His research interests include mechachonics engineering and intelligent manufacturing. 